\title{
Rickettsia bellii sp. nov.: a Tick-Borne Rickettsia, Widely Distributed in the United States, That Is Distinct from the Spotted Fever and Typhus Biogroups
}

\author{
R. N. PHILIP,* E. A. CASPER, R. L. ANACKER, J. CORY, S. F. HAYES, W. BURGDORFER, AND C. \\ E. YUNKER \\ National Institute of Allergy and Infectious Diseases, Rocky Mountain Laboratories, Hamilton, Montana \\ 59840
}

\begin{abstract}
During studies to serotype tick-borne rickettsiae from various areas in the United States, particularly those areas associated with the vectors of Rickettsia rickettsii, we obtained 263 isolates of a rickettsia whose prototype is referred to as strain 369-C. Although rickettsiae of the strain 369-C serotype are distinct from rickettsiae of other serotypes, as determined by cross-micro-immunofluorescence tests of mouse antisera, they do possess one or more antigens that react with convalescent sera from Rocky Mountain spotted fever, epidemic typhus, and murine typhus patients. Because strain 369-C-like isolates have been recovered from eight species of argasid and ixodid ticks from widely separated areas of the United States, we set out to characterize these organisms more precisely, particularly with regard to their relationships to rickettsiae of the spotted fever and typhus groups. The characteristics which we used included morphology as determined by light microscopy and electron microscopy; cytopathogenicity in Vero cell cultures; pathogenicity for embryonated chicken eggs, laboratory mice, guinea pigs, and voles; protein composition as determined by sodium dodecyl sulfate-polyacrylamide gel electrophoresis; and deoxyribonucleic acid base composition. Our results indicated that strain 369-C-like rickettsiae are significantly different phenotypically from rickettsiae of both the spotted fever and typhus groups. Furthermore, the guanine-plus-cytosine content of the deoxyribonucleic acid of strain $369-\mathrm{C}(30 \mathrm{~mol} \%)$ was not different from the guanine-plus-cytosine content of rickettsiae of the typhus group, but it was lower than the guanine-pluscytosine content recorded by other workers for rickettsiae of the spotted fever group. We propose that this species be named Rickettsia bellii sp. nov., with strain RML $369-\mathrm{C}$ as the type strain; all serologically related rickettsiae should henceforth be referred to as the $R$. bellii serogroup.
\end{abstract}

A rickettsia designated strain $369-\mathrm{C}$ was isolated in embryonated chicken eggs from a triturated pool of unfed adult Dermacentor variabilis ticks collected from vegetation near Fayetteville, Ark., on 8 June 1966 (E. J. Bell, Hamilton, Mont., unpublished data). After the fifth serial yolk sac passage, this organism regularly killed chicken embryos 4 to 5 days after inoculation, a characteristic of many tick-borne rickettsiae of the spotted fever group (SFG) (18). Egg-adapted rickettsiae were nonpathogenic or only slightly pathogenic for guinea pigs, inducing fever for 1 to 2 days in one-third of the animals injected but no scrotal reaction and no immunity to challenge by virulent Rickettsia rickettsii. Furthermore, inoculated animals did not develop complementfixing or toxin-neutralizing antibodies against rickettsiae of the SFG and typhus group (TG).

Subsequently, it was shown that strain 369-C stained with fluorescein isothiocyanate-conju- gated $R$. rickettsii antibody raised in rabbits, which was reactive to varying degrees against all members of the SFG (13). When mouse antisera were used in a micro-immunofluorescence (micro-IF) serotyping test (13), strain 369-C was shown to be distinct from 72 other strains in 12 SFG and 3 TG serotypes from the Eastern and Western Hemispheres. Because at that time strain $369-\mathrm{C}$ was the only representative, its assignment to a new serotype was reserved pending further evidence of the existence of similar strains in nature. Since 1977, many rickettsial isolates serologically resembling strain 369-C have been recovered from ticks collected in widely separated localities in the United States. Various biological characteristics of representative strains are described in this paper. A new species name is proposed for this rickettsia, and its relationship to rickettsiae of the SFG and TG, particularly $R$. rickettsii, is discussed. 


\section{MATERIALS AND METHODS}

All isolates were characterized for morphology by light microscopy, cytopathogenicity in Vero cell cultures, and serotype by micro-IF tests of mouse antisera (13). Other biological properties were examined for the 22 strains from various tick species and geographic sources listed in Table 1. The characteristics studied for one or more of the strains listed included pathogenicity for chicken embryos, randomly bred adult white Swiss mice (Rocky Mountain Laboratory strain), guinea pigs, and laboratory-reared voles (Microtus pennsylvanicus); serological cross-reactivity of convalescent sera to rickettsial infections in humans and other mammals; electron microscopic morphology; protein composition as determined by sodium dodecyl sulfatepolyacrylamide gel electrophoresis (SDS-PAGE); and deoxyribonucleic acid (DNA) base composition.

Detection of rickettsia-like organisms in systemically infected ticks was accomplished by the hemolymph test (HT) of Burgdorfer (2), which was modified as previously described $(12,15)$. Isolation of rickettsiae from HT-positive ticks in Vero cell monolayers was done by the method of Cory et al. (3). Two or three serial passages of isolates in Vero cells were necessary before the concentration of organisms was sufficient for typing with mouse antisera (13). The procedures used to determine the pathogenicities of isolates for chicken embryos, guinea pigs, and voles have been described previously $(12,15)$.

For electron microscopic studies, Vero cells infected with strain 369-C or strain SH-130 (strain 3 [Table 1]) were harvested at $120 \mathrm{~h}$. The cells were sedimented by centrifugation at $450 \times g$, and the resulting pellets were washed once in tissue culture maintenance medium L-15 (3) before they were suspended in L-15 medium containing either $10 \%$ normal rabbit serum or $10 \%$ rabbit antiserum against strain $\mathrm{SH}-130$. The cells in a portion of each sample were then mechanically disrupted by the procedure of Silverman et al. (17) to release intracellular rickettsiae and to stabilize the external coat (slime layer) with antibody. The preparations were then fixed and processed for examination with a Hitachi model HU-11E electron microscope at $75 \mathrm{kV}(4)$.

The proteins of representative isolates were determined by SDS-PAGE in $9 \%$ polyacrylamide slab gels, using the method of Laemmli (6). Rickettsiae were purified from Vero cell cultures by Renografin density gradient centrifugation (20). Whole cells $(1.0 \mathrm{mg})$ were solubilized in $0.1 \mathrm{ml}$ of sample buffer by boiling for 6 $\mathrm{min}$, and $15 \mu \mathrm{l}$ of solubilized protein was applied to each gel.

DNA guanine-plus-cytosine $(\mathrm{G}+\mathrm{C})$ base ratios were determined by the method of Tyeryar et al. (19), as described by Anacker et al. (1).

\section{RESULTS}

Since 1974, 263 isolates of the strain 369-C serotype have been identified by micro-IF (Table 2). Strain 369-C-like rickettsiae have been detected in ticks from all geographic regions of the United States from which ticks have been examined. A total of 23 countries in seven states are represented, and in many localities strain
369-C was the predominant serological form among the isolates obtained. Major surveys for rickettsiae which were systemically infecting ticks were conducted in Montana, California, North Carolina, and Ohio. Rickettsiae of the strain 369-C serotype comprised $41(39 \%)$ of 106 isolates from Dermacentor andersoni in Montana, $19(13 \%)$ of 149 isolates from California, 59 $(82 \%)$ of 72 isolates from North Carolina, and $128(83 \%)$ of 154 isolates from Ohio.

The strain 369-C serotype has been identified in eight species of ixodid and argasid ticks representing four genera (Table 2). Two species (D. andersoni and D. variabilis) are the principal vectors of $R$. rickettsii to humans, Dermacentor occidentalis is thought to transmit spotted feve. in California (7), and Haemaphysalis leporispalustris may be important in the natural maintenance of $R$. rickettsii; however, four tick species (Dermacentor parumapertus, Dermacentor albipictus, Argas cooleyi, and Ornithodoros concanensis) have not been incriminated either in the transmission or in the ecology of $R$. rickettsii.

Serological reaction patterns. Little or no cross-reaction of isolates of the strain 369-C serotype with rickettsiae of other serotypes was evident in micro-IF tests of mouse typing antisera. Serological heterogeneity was observed among strains of the strain 369-C serotype from various tick species (Table 3 ). Thus, the prototype (strain 369-C; serial no. 1) cross-reacted poorly with strain CA-424 from $D$. parumapertus (serial no. 4) and did not react at all with the two strains from argasid ticks, $A$. cooleyi and $O$. concanensis (serial no. 7 and 8). On the other hand, strain SH-130 from D. andersoni (serial no. 2) cross-reacted well with strain 369-C, strain CA-424, and both argasid tick strains. That this variability among strains was not strictly an association with the tick species of origin was shown by the spectrum of crossreactivity between strain $369-\mathrm{C}$ and the Ohio isolates, all of which were from $D$. variabilis. Strain 0-2006 (strain 7 [Table 1]) cross-reacted broadly with strain 369 -C-like rickettsiae from all tick species (data not shown). Nevertheless, within given geographic regions, isolates from $D$. andersoni or $D$. variabilis were remarkably uniform in their spectrum of reactivity and also in their characteristics of cytopathogenicity (see below).

Despite their distinctness from other rickettsial serotypes, strain 369-C-like isolates did share with members of the SFG one or more similar or identical antigens to which our randomly bred Swiss mice did not respond. This was suggested early in our study when these organisms were detected during HT surveys in tick hemocytes by direct immunofluorescence with rabbit anti$R$. rickettsii conjugate, and all strain 369-C-like 


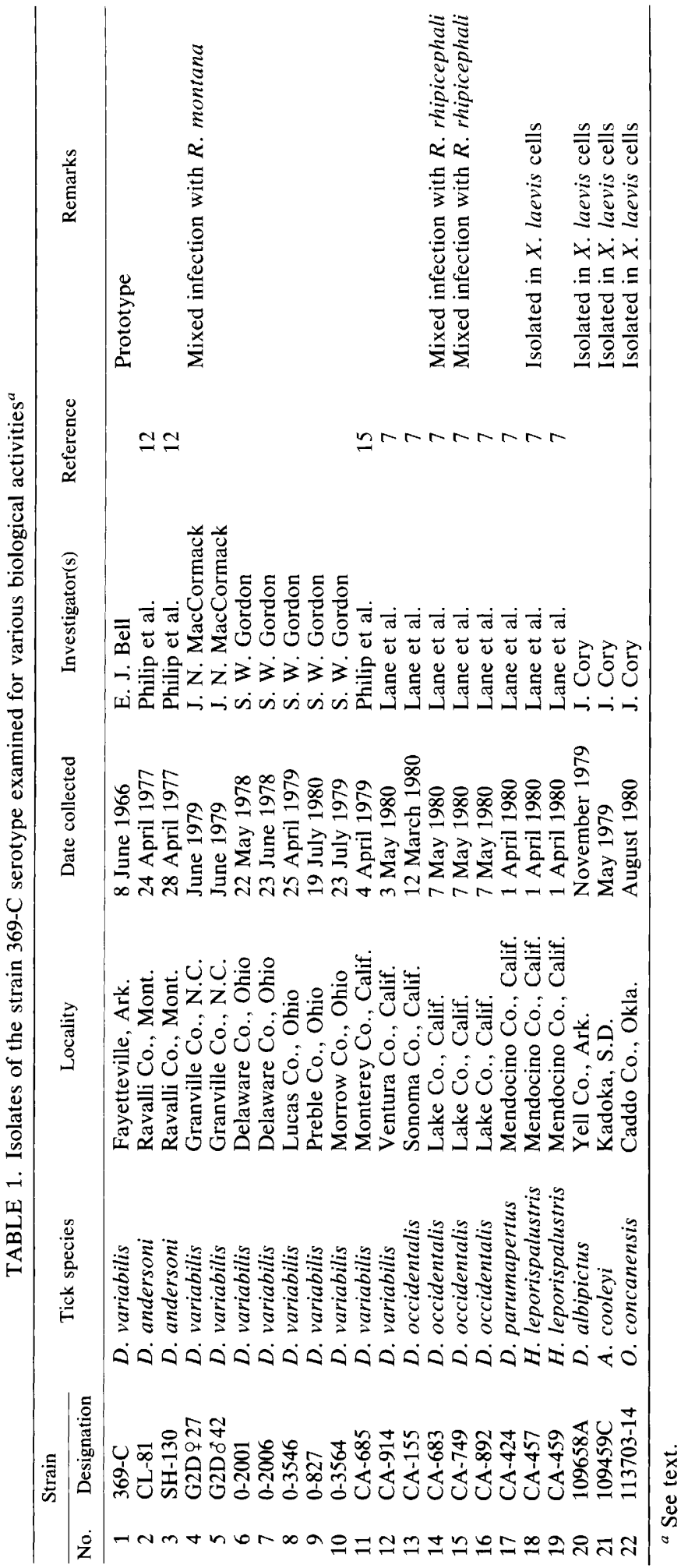


isolates stained to varying degrees with this conjugate.

Morphology. Although three isolates of the strain 369-C serotype were recovered from ticks (D. variabilis and $H$. leporispalustris) that were HT negative, isolation was usually successful only from ticks containing red-staining (Giménez stain), often delicate, hemocyte-associated, rod-shaped cells measuring about 0.3 to 0.4 by 2.0 to $3.0 \mu \mathrm{m}$ (Fig. 1a). Strain 369-C-like isolates were never recovered from ticks containing the lanceolate, coccobacillary cells that are morphologically typical of $R$. rickettsii, Rickettsia montana, and Rickettsia rhipicephali, nor were the strain 369-C-like isolates easily confused with the large, bacillary, basophilic-staining, bacterium-like organisms referred to as "long forms" that do not stain with fluorescein isothiocyanate-conjugated SFG antibody and are often present in hemocytes of $D$. andersoni and $D$. variabilis (2). The ultrastructure of strain $369-\mathrm{C}$ resembles that reported for $R$. rickettsii (16) in many respects (Fig. 2). Organisms in situ in Vero cells are surrounded by a nominal halozone (Fig. 2A through C), which appeared to be a slime layer when the rickettsiae were released into a menstruum containing homotypic antibody (Fig. 2B and D). Strain 369-C has a prominent trilaminar cell wall consisting of an inner leaflet that is approximately twice as thick as the outer leaflet (Fig. 2C and D). The splotchy, fibrillar cytoplasm containing evenly distributed ribosomes is enclosed within a plasma membrane (Fig. 2C). The major differences between strain 369-C and $R$. rickettsii are the greater length-versus-width ratio of the former and the absence in strain 369-C of a conspicuous microcapsular layer immediately external to the cell wall (Fig. 2C). Although replication of strain 369-C occurs by binary fission principally within the cytoplasm of host cells, intranuclear growth is observed occasionally (Fig. 2A).

Plaque morphology and behavior in cell culture. Vero cell culture was usually a satisfactory system for primary recovery of rickettsiae of the strain 369-C serotype from HT-positive ticks. Thus, isolation was successful from $86 \%$ of the $D$. andersoni individuals having hemocyte-associated organisms morphologically resembling strain 369-C in the Montana survey and from $73 \%$ of the North Carolina and $52 \%$ of the Ohio HT-positive $D$, variabilis individuals. In the last instance, the ticks had been stored at $-70^{\circ} \mathrm{C}$ for 6 months to 2.5 years before they were processed. However, in California, $48 \%$ of the 190 $H$. leporispalustris individuals removed from five Lepus californicus were HT positive for organisms morphologically similar to strain 369 $\mathrm{C}$, yet only four isolations from these ticks were obtained in Vero cells. That Vero cells were not optimal for primary isolation in this instance was suggested by the recovery of a strain 369-C-like isolate (strain 18 [Table 1]) in the poikilothermic Xenopus laevis (South African clawed toad) cell line when simultaneously inoculated Vero cell monolayers were negative (Cory et al., manuscript in preparation).

Variations in the susceptibility of Vero cells to primary infection were also shown by differences in the nature of cytopathogenicity according to locality and arthropod host. All strain 369C-like isolates from $D$. andersoni in western Montana gave characteristic and well-defined but faint ring-shaped plaques. A similar plaque morphology was observed for all isolates from $D$. variabilis from Ohio and from $D$. variabilis and $D$. occidentalis from California. On the other hand, distinct plaques were not formed in Vero cells by isolates from North Carolina, and little cytopathic effect could be demonstrated for isolates obtained from $H$. leporispalustris, $D$. albipictus, $A$. cooleyi, and $O$. concanensis even though all cells were intensely infected. In every instance, strain 369-C-like isolates showed little lethal effect on the Vero host cells until cellular integrity was compromised by the rickettsial burden. The yields of Vero cell-cultivated, purified rickettsiae of the strain 369-C serotype were

TABLE 2. Isolates of the strain 369-C serotype by arthropod source according to state

\begin{tabular}{|c|c|c|c|c|c|c|c|c|}
\hline \multirow{3}{*}{ State } & \multicolumn{8}{|c|}{ No. of isolates from: } \\
\hline & \multicolumn{6}{|c|}{ Ixodid ticks } & \multicolumn{2}{|c|}{ Argasid ticks } \\
\hline & $\begin{array}{c}D \\
\text { variabilis }\end{array}$ & $\begin{array}{c}D . \\
\text { andersoni }\end{array}$ & $\begin{array}{c}D . \\
\text { occidentalis }\end{array}$ & $\begin{array}{c}D . \\
\text { parumapertus }\end{array}$ & $\begin{array}{c}D . \\
\text { albipictus }\end{array}$ & $\begin{array}{l}\text { H. leporis- } \\
\text { palustris }\end{array}$ & $\begin{array}{c}\text { A. } \\
\text { cooleyi }\end{array}$ & $\begin{array}{c}O . \\
\text { concanensis }\end{array}$ \\
\hline Arkansas & $1(1)^{a}$ & & & & $1(1)$ & & & \\
\hline California & $10(2)$ & & $4(2)$ & $1(1)$ & & $5(1)$ & & \\
\hline Montana & & $51(2)$ & & & & & & \\
\hline North Carolina & $60 \quad(1)$ & & & & & & & \\
\hline Ohio & $128(11)$ & & & & & & & \\
\hline Oklahoma & & & & & & & & $1(1)$ \\
\hline South Dakota & & & & & & & $1(1)$ & \\
\hline
\end{tabular}

${ }^{a}$ The numbers in parentheses are the numbers of counties from which ticks were obtained. 


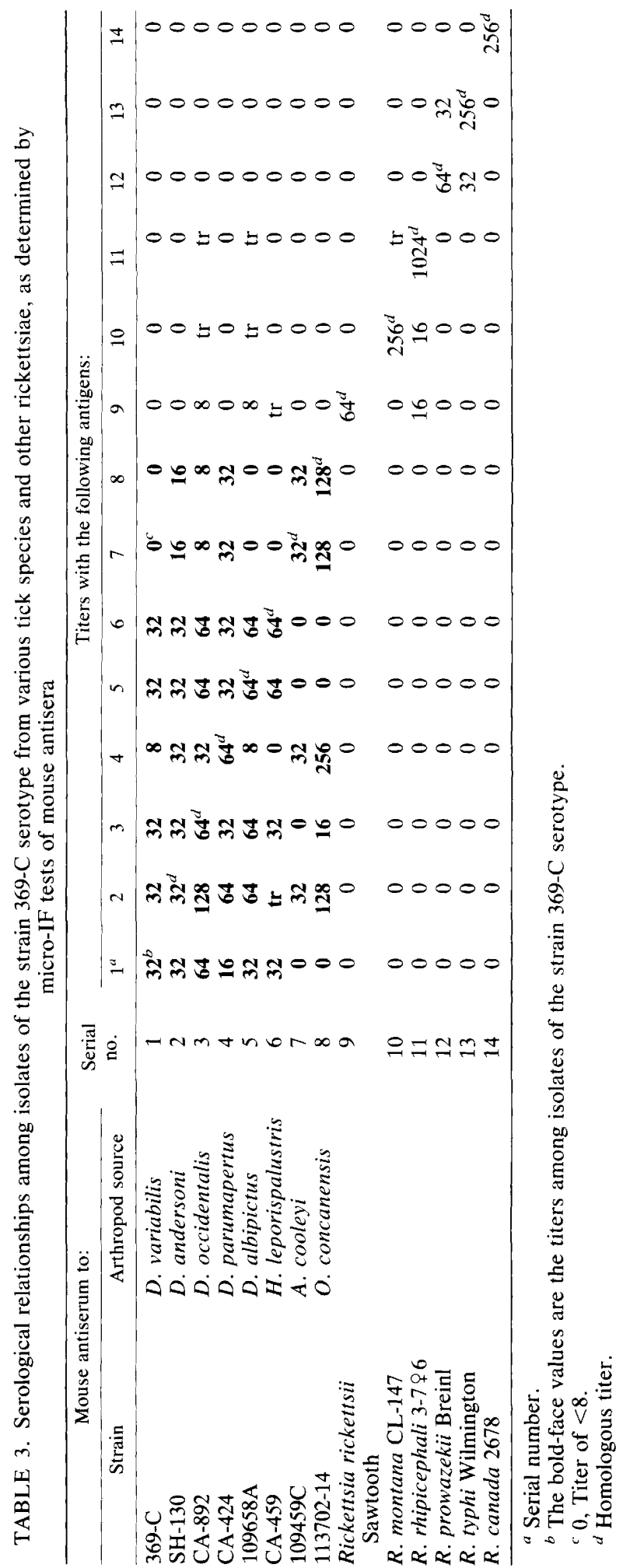



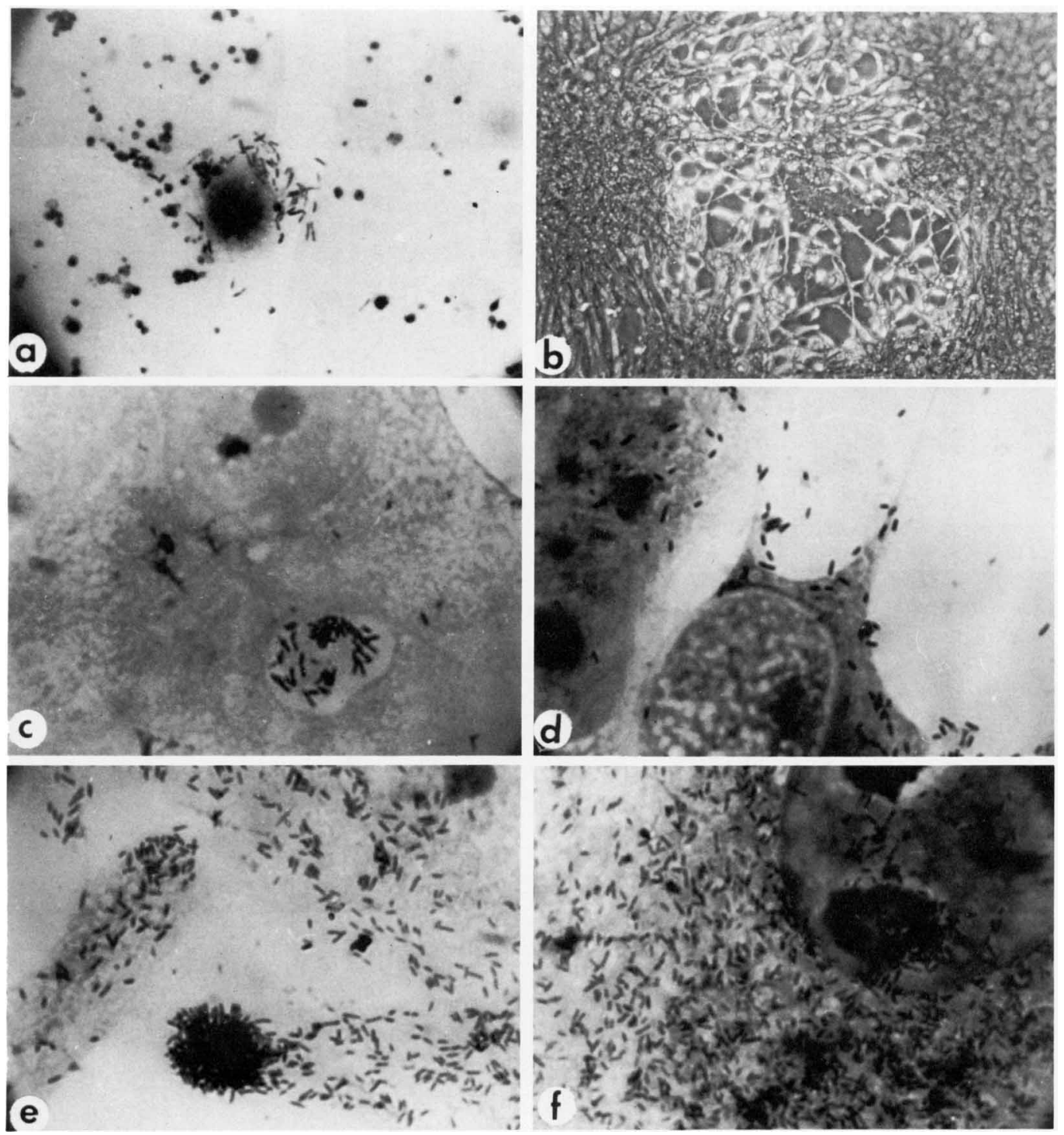

FIG, 1. (a) Hemocyte from $D$. andersoni containing delicate, red-staining bacilli. The strain 369-C-like isolate strain SH-130 was isolated from this tick. Giménez stain. $\times 2,700$. (b) Typical astrocytic plaque morphology induced in Vero cells by rickettsiae of the strain 369-C serotype. Strain CA-914 from D. variabilis on day 4 (primary passage). Unstained. $\times 255$. (c) Strain $\mathrm{SH}-130$ rickettsiae in an irradiated Vero cell $24 \mathrm{~h}$ after infection. Organisms were usually discretely scattered throughout the cytoplasm, but occasionally rickettsia-containing cyst like structures without a limiting membrane were observed. Giménez stain. $\times 2,700$. (d) Strain SH-130 rickettsiae in an irradiated Vero cell $48 \mathrm{~h}$ after infection. The cell processes which often appeared to serve as intercellular bridges are prominent. Giménez stain. $\times 2,700$. (e) Strain SH-130 rickettsiae in an irradiated Vero cell $72 \mathrm{~h}$ after infection. Numerous organisms are present in the cell processes. Retraction of a process sometimes led to a terminal "blossom" consisting of a mass of organisms. Giménez stain. $\times 2,700$. (f) Strain SH130 rickettsiae in an irradiated Vero cell $96 \mathrm{~h}$ after infection. Despite the heavy rickettsial burden, the integrity of the host cell does not appear to be compromised. Giménez stain $\times 2,700$.

5- to 10 -fold higher than the yields of the SFG serotypes $(R$. rickettsii, $R$. montana, and $R$. rhipicephali), which exhibited greater cytopathogenicity.
Vero cell monolayers infected with plaqueforming strains of strain 369-C-like rickettsiae first showed macroscopic cytopathic changes at about $72 \mathrm{~h}$ after inoculation (usually 6 to $12 \mathrm{~h}$ 

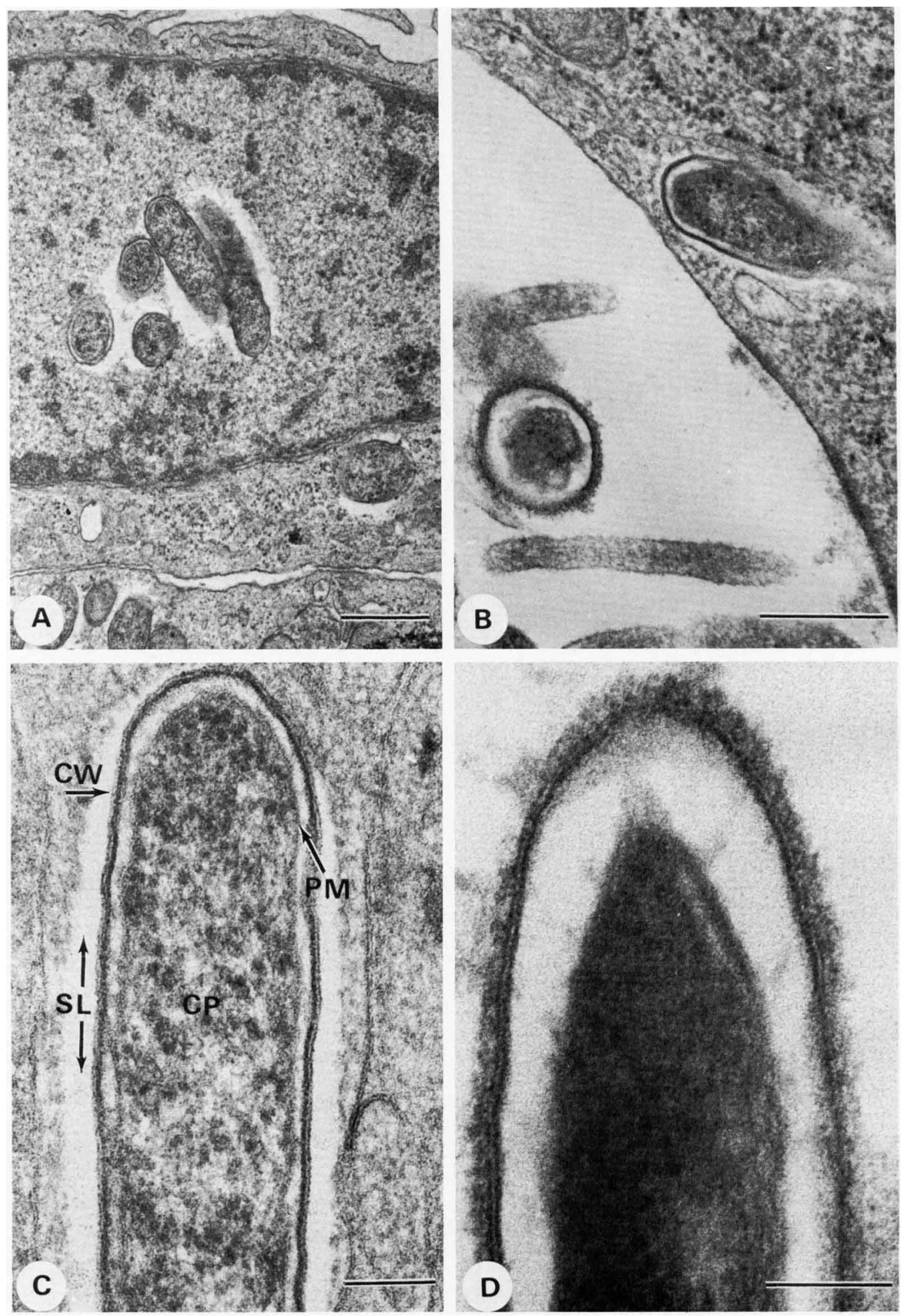

FIG. 2. Electron micrographs of strain 369-C rickettsiae. (A) Rickettsiae in the nucleus of a Vero cell. Bar = $0.5 \mu \mathrm{m}$. (B) Rickettsiae intra- and extracellularly in a Vero cell culture. The extracellular organism is bound by antibody. Bar $=0.3 \mu \mathrm{m}$. (C) Micrograph showing the slime layer (SL) and ultrastructural details of the cell wall $(\mathrm{CW})$. plasma membrane $(\mathrm{PM})$, and cytoplasm $(\mathrm{CP})$. Bar $=0.1 \mu \mathrm{m}$. (D) Micrograph showing ultrastructural detail of an extracellular organism bound by antibody. Bar $=0.1 \mu \mathrm{m}$. 
earlier than such changes induced by $R$. rickettsii). Minute foci of refractile spider-like cells developed (Fig. 1b), and these characteristic plaques enlarged by spreading centrifugally over ensuing days. The centers of evolving plaques consisted of Vero cells that had resumed normal architecture and stained intensely with neutral red, thus accounting for a ring-shaped appearance after 5 to 6 days.

The growth characteristics of a plaque-forming strain 369-C-like isolate (strain SH-130) were determined in slide chamber cultures of irradiated Vero cells by light microscopy, using the method of Wisseman et al. (21). Rickettsiae were inoculated onto monolayers at a concentration that resulted after $2 \mathrm{~h}$ in infection of about $80 \%$ of the cells with an average of three organisms per cell. By $24 \mathrm{~h}$ the rickettsiae in some cells were numerous (Fig. 1c), and by $96 \mathrm{~h}$ the cytoplasm of all cells was massively packed with rickettsiae (Fig. 1f). The first effect observed on host cells after 36 to $48 \mathrm{~h}$ was a change to astrocytic morphology, with the appearance of numerous processes that often appeared to interconnect neighboring cells (Fig. 1d). Rickettsiae were usually present in these cellular bridges; conceivably, the rickettsiae were transferred from infected cells to noninfected cells by this mechanism (Fig. 1e). This would account for the centrifugal spread of infection, particularly since few extracellular organisms were observed until the late stages.

Pathogenicity for experimental animals. (i) Mice. The Swiss white mice used in the preparation of antisera showed no evidence of illness after inoculation with any strain 369-C-like isolate. The homologous micro-IF antibody titers 10 days after injection ( 32 to 256 ) were generally lower than the titers obtained with SFG rickettsiae $(256$ to 32,768$)$, suggesting that replication of strain 369-C-like rickettsiae was slight, if it occurred at all. Pathogenicity for suckling mice was not determined.

(ii) Embryonated eggs. Although prototype strain 369-C was isolated and established in embryonated eggs by yolk sac passage, most strain 369-C-like isolates tested were nonpathogenic for chicken embryos. Attempts to establish 17 of the strains listed in Table 1 in embryonated eggs were successful in only three instances. After two to four serial yolk sac passages, strains 5,21 , and 22 killed embryos regularly between days 4 and 8 postinoculation. Replication of rickettsiae was poor; the concentrations of the organisms in yolk sacs never exceeded more than a few organisms per $\times 1,000$ microscopic oil immersion field. Five Ohio strains (strains 6 through 10 [Table 1]) killed embryos sporadically, and a few organisms were observed after five serial passages, which indi- cated limited multiplication and pathogenicity for chicken embryos. The Montana and California strains could not be passed serially in chicken embryo yolk sacs.

(iii) Guinea pigs. Intraabdominal inoculation of viable cell culture preparations of 21 isolates of the strains $369-\mathrm{C}$ serotype were essentially nonpathogenic for guinea pigs and seldom gave rise to even low ( 8 to 256 ) homologous micro-IF antibody titers. Animals in groups of six were inoculated with graded doses ( 1 to $6 \log _{10}$ Vero cell plaque-forming units) of strains CL-81 (strain 2 [Table 1]) and SH-130, and these animals were fully susceptible to challenge with virulent $R$. rickettsii 14 days later; however, a few also showed anamnestic antibody responses to the strain used for primary inoculation. Since replication of strain 369-C-like isolates did not occur in this animal or was minimal at best, the immunological cross-relationships of these organisms to $R$. rickettsii could not be determined.

(iv) $M$. pennsylvanicus. Rickettsiae of the strain 369-C serotype caused neither death nor illness in laboratory-reared voles. Isolates usually induced low micro-IF antibody responses, and a few organisms were observed in smears of tunicas in about one-half of the animals examined on day 5. Rickettsiae could not be recovered after subpassage of tunicas and spleens to noninfected voles, and antibody conversion after secondary passage was infrequent.

In parallel attempts to detect rickettsiae in North Carolina ticks, preparations from HTpositive $D$. variabilis individuals were inoculated simultaneously into voles and Vero cells. Of 60 tick preparations from which strain 369-C-like isolates were obtained in Vero cells, 58 also gave rise in voles to antibodies directed principally against the strain 369-C serotype (titers, 32 or 64). Of 14 ticks for which isolation attempts were unsuccessful, 3 nevertheless induced antibodies to strain 369-C. Likewise, ticks infected with other types of rickettsiae $(R$. rickettsii and $R$. montana) stimulated antibody responses in voles specific for the serotype isolated. Thus, inoculation of ticks directly into voles seemed to be a sensitive method for detecting and identifying the infecting organism in a one-way test. These results also reassured us that the serological nature of the rickettsiae did not change during the two to three Vero cell passages necessary before the isolates could be serotyped by cross-tests of mouse antisera.

Serological responses in other species. By one definition (5), rickettsiae are classified serologically as members of the SFG on basis that they possess one or more major antigens that crossreact with $R$. rickettsii antiserum. However, laboratory mice are serologically unresponsive to the SFG-reactive antigen(s) (13), and antisera 


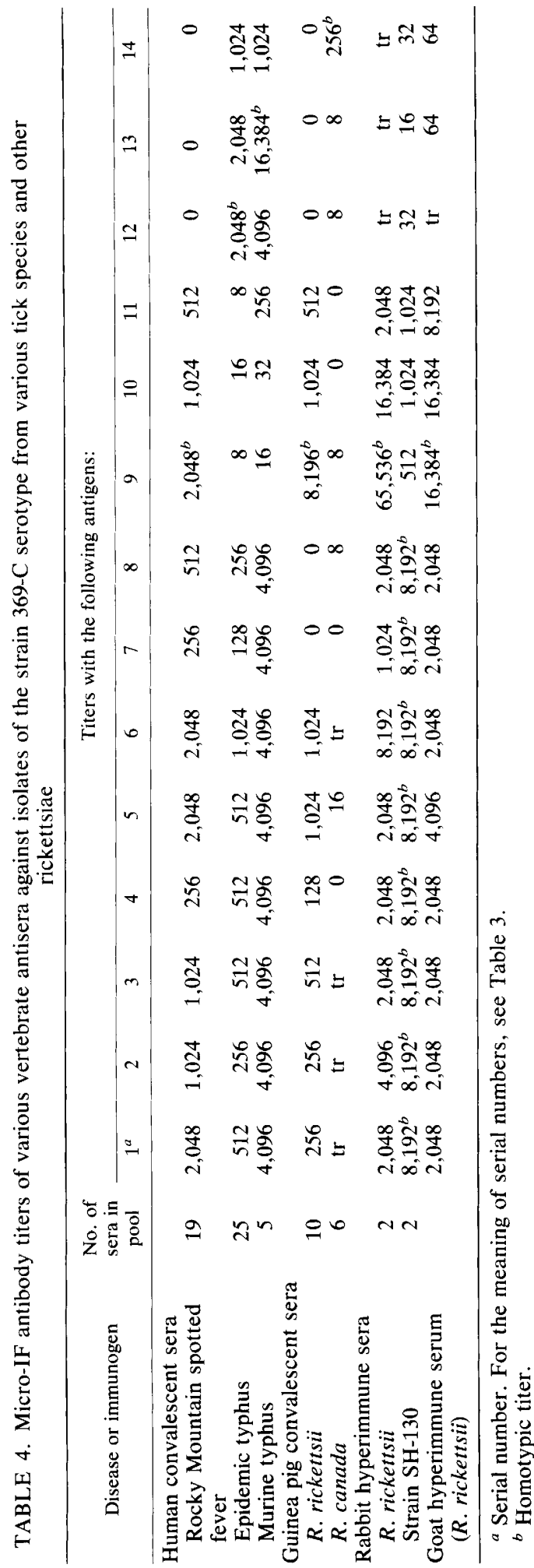

from animal species such as guinea pigs, rabbits, and humans must be used to demonstrate group relationships. The fact that all strain 369-C-like isolates stained with fluorescein isothiocyanateconjugated rabbit $R$. rickettsii antiserum provided evidence that the strain 369-C serotype belonged to the SFG. However, it was surprising to discover later that minimal staining of some isolates also occurred with our conjugated rabbit antiserum to Rickettsia prowazekii.

For the past 3 years we have regularly tested sera from suspect cases of Rocky Mountain spotted fever by micro-IF (14) against the strain 369-C-like isolate strain $\mathrm{SH}-130$, as well as against a variety of SFG and TG antigens. Patients showing antibody responses to $R$. rickettsii consistently also developed cross-reacting responses to strain $\mathrm{SH}-130$.

Strains of the strain 369-C serotype share at least one similar or identical antigen with both the SFG and the TG. Table 4 shows the patterns of cross-reactivity in pooled sera from humans and other mammals infected or immunized with $R$. rickettsii or TG rickettsiae against the strain 369-C-like isolates from eight tick species listed in Table 3. The convalescent serum pool from 19 serologically confirmed (increase in titer to $R$. rickettsii of fourfold or more) Rocky Mountain spotted fever patients from Montana and North Carolina had a homologous titer of 2048; the heterologous titers to other SFG rickettsiae and the strain 369 -C-like isolates were one- to eightfold lower than the homologous titer. The sera were nonreactive against the TG. Cross-reactivity was both of the immunoglobulin $\mathrm{M}$ class and the immunoglobulin $\mathrm{G}$ class (data not shown).

Likewise, a convalescent serum pool from serologically confirmed Ethiopian epidemic typhus patients showed strong cross-reactions against the strain 369-C-like isolates but very little reactivity against SFG rickettsiae. The same was true of sera from murine typhus patients from Texas and North Carolina. The cross-reacting strain $369-\mathrm{C}$ antibodies in the typhus sera were almost entirely of the immunoglobulin M class (data not shown).

Guinea pigs inoculated with $R$. rickettsii developed cross-reacting antibodies to all strain 369-C-like isolates except those from argasid ticks. Guinea pigs inoculated with Rickettsia canada developed very little cross-reactivity to strain 369-C-like isolates. Rabbits and a goat hyperimmunized with $R$. rickettsii developed strong cross-reacting antibodies against all strain 369-C-like isolates but very little reactivity to TG rickettsiae. Finally, sera from rabbits hyperimmunized to strain SH-130 reacted substantially against SFG rickettsiae but showed little or no cross-reactivity to TG rickettsiae. 
SDS-PAGE. Because the serological reactivity of certain components of strain 369-C was shared with both the SFG and the TG rickettsiae and because there appeared to be some diversity in several biological characteristics among the strain 369-C-like isolates, the protein constituents of these organisms were examined by SDSPAGE (Fig. 3).

The migration patterns of the solubilized proteins derived from two strain 369-C-like isolates (Fig. 3A, lanes 11 and 12) were sharply different from those of Proteus OX-19 (lane 1) and three TG (lanes 2 through 4) and six SFG (lanes 5 through 10) rickettsiae. Proteus OX-19 (American Type Culture Collection) was included because it possesses an antigen that reacts with early convalescent sera from TG and some SFG illnesses. The major bands of strain 369-C-like isolates did not correspond to the major bands of the other rickettsiae, and, in fact, the similarities

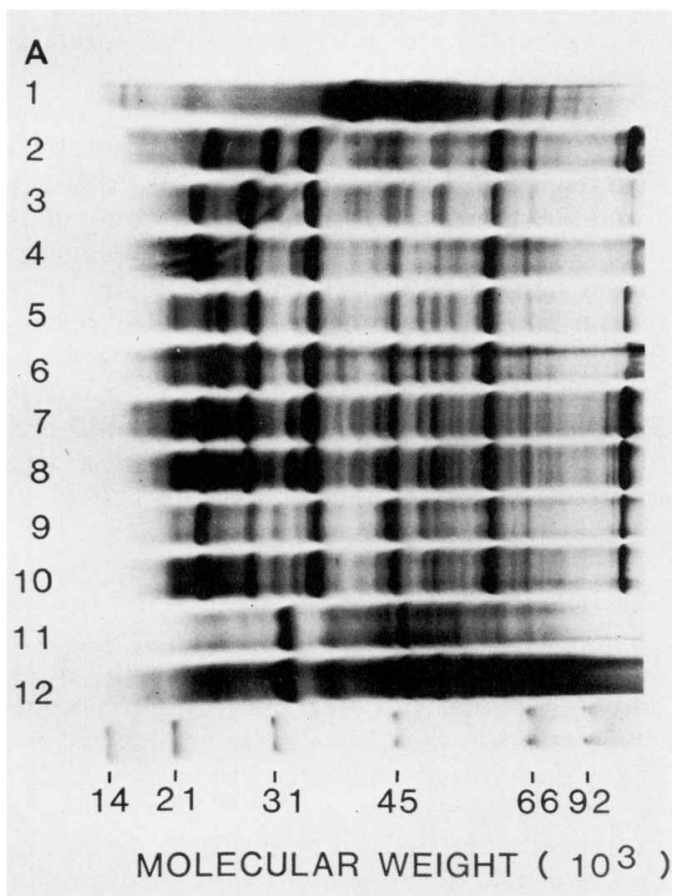

in electrophoretic patterns between TG and SFG strains were greater than the similarities between either group and strain 369-C.

There was considerable variation in the protein profiles of the 369-C-like isolates derived from eight tick species, as determined by SDSPAGE (Fig. 3B), but there were areas of similarity in the electrophoretic patterns which were common to all strains, including a major protein with a molecular weight of 33,000 and lesser components with molecular weights of 45,000 and 48,000 . The 33,000-dalton band may be dimeric and dependent on the conditions used for solubilizing the rickettsiae. When dithiothreitol was incorporated into the sample buffer, a faster-moving component was often observed at a molecular weight of 30,000 (Fig. 3B, lanes 3

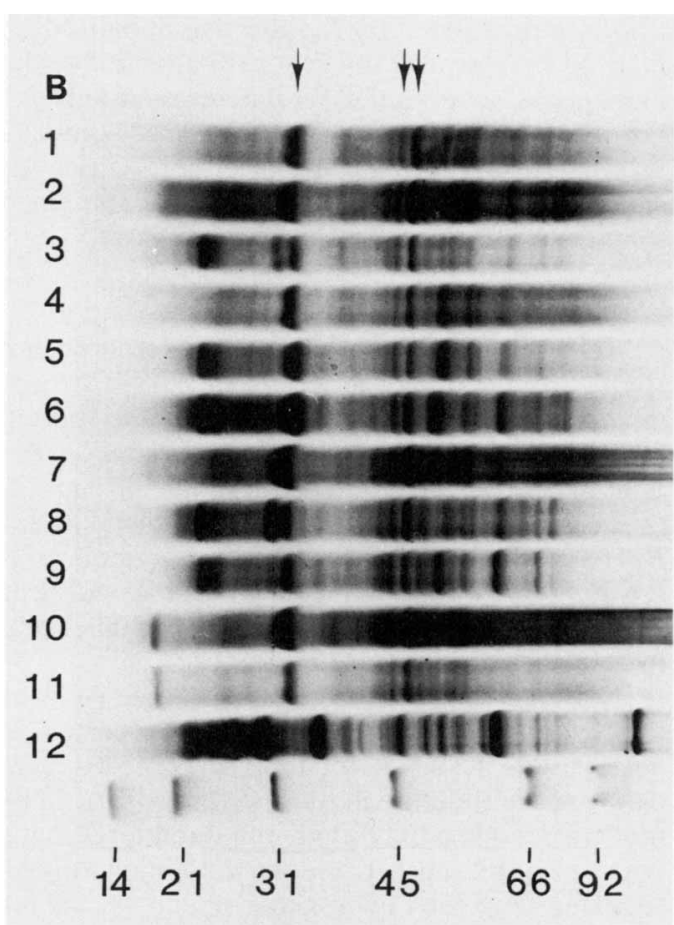

MOLECULAR WEIGHT $\left(10^{3}\right)$

FIG. 3. (A) SDS-PAGE of Proteus OX-19 and strains of rickettsiae representing various serotypes. Lane 1, Proteus OX-19; lane 2, $R$. prowazekii Breinl; lane 3, $R$. typhi Wilmington; lane 4, $R$. canada 2678; lane 5, $R$. rickettsii $\mathrm{R}$; lane $6, R$. rickettsii Sawtooth; lane $7, R$. montana $\mathrm{M} / 5-6$; lane $8, R$. montana P1-51; lane $9, R$. rhipicephali 3-7 9 6; lane 10, $R$. rhipicephali CL-146; lane 11, strain 369-C; lane 12, strain SH-130. (B) SDS-PAGE of strains of the strain 369-C serotype from various tick species and geographic areas. Lane 1, strain 369-C from $D$. variabilis from Arkansas; lane 2, strain 0-2006 from $D$. variabilis from Ohio; lane 3, strain G2D $\delta 42$ from $D$. variabilis from North Carolina; lane 4, strain SH-130 from D. andersoni from Montana; lane 5, strain CL-81 from D. andersoni from Montana; lane 6, strain CA-892 from D. occidentalis from California; lane 7, strain 109658A from D. albipictus from Arkansas; lane 8. strain CA-424 from D. parumapertus from California; lane 9, strain CA-459 from $H$. leporispalustris from California; lane 10, strain $109459 \mathrm{C}$ from A. cooleyi from South Dakota; lane 11 , strain 113704-14 from $O$. concanensis from Oklahoma; lane $12, R$. rickettsii $\mathrm{R}$ from $D$. andersoni from Montana. The arrows indicate bands of identity common to the strain 369-C-like isolates. 
and 8). As might be expected, the greatest differences in electrophoretic pattern appeared to be between the argasid tick strains (Fig. 3B, lanes 10 and 11) and the ixodid tick strains (lanes 1 through 9). Only slight variations in pattern were evident in strains from the same tick species from different geographic areas (e.g., $D$. variabilis strains from Arkansas, Ohio, and North Carolina) (Fig. 3B, lanes 1 through 3).

DNA base composition. Serologically, the agents of the strain 369-C serotype seemed to be related to the $\mathrm{SFG}$, but there were enough differences in morphology, pathogenicity, and degree of serological relatedness, as well as the low-grade cross-reactivity to the $\mathrm{TG}$, to suggest that the strain 369-C-like isolates may not be true SFG rickettsiae. To gain more precise information on the taxonomic position of these agents, we determined the DNA base composition of strain $369-\mathrm{C}$ by the thermal denaturation method of Marmur and Doty (8) (Table 5). The mean $\mathrm{G}+\mathrm{C}$ content in four determinations for the strain $369-\mathrm{C}$ prototype $(30.1 \pm 0.4 \mathrm{~mol} \%)$ was similar to the values reported for TG rickettsiae $(10,19)$ but lower than the values obtained for SFG rickettsiae (about $32.5 \mathrm{~mol} \%$ ) $(1,19)$.

\section{DISCUSSION}

Rickettsiae of the strain $369-\mathrm{C}$ serotype are frequently encountered in two or possibly three major tick vectors of Rocky Mountain spotted fever throughout their geographical ranges in the United States. Strain 369-C systemically infects these vectors and not only may be mistaken for $R$. rickettsii in the HT but also, more importantly, may be involved in the ecology of this pathogen in some as-yet-undefined manner and may influence its focal distribution.

Early on, it was thought that strain $369-\mathrm{C}$ was a rickettsia of the SFG, based on its association with ixodid ticks, its apparent strict cell dependency for replication in these arthropods, and its modest staining by direct immunofluorescence with a rabbit anti- $R$. rickettsii conjugate. As reported here, however, other phenotypic characteristics of strain 369-C and related organisms distinctly set them apart from the SFG. Thus, they could be sharply differentiated from SFG rickettsiae on the basis of morphology, ultrastructure, serological reactivity, and protein composition. It remained to be demonstrated that strain 369-C was also genetically distinct from rickettsiae of the SFG. The DNA base composition (30 mol\% $\mathrm{G}+\mathrm{C}$ ) differentiated strain 369-C from $R$. rickettsii $(1,19)$, Rickettsia akari (19), $R$. montana (1), and $R$. rhipicephali (1), all of which have $\mathrm{G}+\mathrm{C}$ contents of about $32.5 \mathrm{~mol} \%$. Thus, the hypothesis that strain 369 $\mathrm{C}$ is a variant nonpathogenic or degradation form of $R$. rickettsii is untenable. It is likely that
TABLE 5. G $+\mathrm{C}$ content of strain DNA, 369-C as determined by thermal denaturation temperature

\begin{tabular}{ccc}
\hline Expt & $\begin{array}{c}T_{m} \\
\left({ }^{\circ}\right)^{a}\end{array}$ & $\begin{array}{c}\mathrm{G}+\mathrm{C} \\
\text { content as } \\
\text { determined } \\
\text { by } T_{m} \\
(\mathrm{~mol} \%)^{b}\end{array}$ \\
\hline 1 & & 30.0 \\
2 & 81.6 & 29.5 \\
3 & 81.4 & 30.5 \\
& 81.8 & 30.0 \\
\hline
\end{tabular}

${ }^{a} T_{m}$, Thermal denaturation temperature of DNA. The thermal denaturation temperature of Escherichia coli DNA (included as an internal standard) was $90.1^{\circ} \mathrm{C}$.

${ }^{b}$ Calculated from the formula of Marmur and Doty (8). The mean \pm standard deviation was $30.1 \pm 0.4$ mol\%.

strain 369-C-like organisms, the progenitor of which emerged millennia ago, are phylogenetically distinct.

The DNA base ratio of strain $369-\mathrm{C}$ is not significantly different from the base ratios of $\mathrm{TG}$ rickettsiae and $R$. canada $(10,11,19)$. Although strain $369-C$ possesses an antigenic component that reacts with convalescent sera from epidemic and murine typhus patients and some other mammalian species, there are no other phenotypic characteristics that are shared by strain 369-C and TG rickettsiae. Furthermore, strain 369-C does not resemble $R$. canada (9). Therefore, it is unlikely that this strain is a true member of the TG. However, this should be confirmed by DNA homology studies.

It is known that some strains of strain 369-Clike rickettsiae from western Montana can be transovarially and transstadially transmitted in D. andersoni (Philip, unpublished data; Burgdorfer, unpublished data), but virtually nothing is known about the natural maintenance of these rickettsiae. Based on limited laboratory studies on the pathogenicity of these organisms for experimental animals, it might be surmised that they are maintained primarily by transmission from tick to tick. However, there appears to be some variability in pathogenicity among strains, and the gamut of candidate vertebrate hosts has by no means been examined or defined.

We propose here to recognize these rickettsiae as a new species and to name this species in honor of the rickettsiologist E. John Bell, who isolated and first studied the prototype, strain 369-C. Hence, Rickettsia bellii is hereby proposed as the name for strain 369-C; all strains with similar serological characteristics should henceforth be referred to as members of the $R$. bellii serogroup. The distinguishing biological 
characteristics of $R$. bellii and the $R$. bellii serogroup are given in the following description.

Description of Rickettsia bellii sp. nov. Rods 0.3 to 0.4 by 2.0 to $3.0 \mu \mathrm{m}$ during the $\log$ phase of growth, sometimes 10.0 to $15.0 \mu \mathrm{m}$ under suboptimal conditions of tissue culture. Cells possess an outer slime layer and a trilaminar cell wall with the inner leaflet about twice as thick as the outer leaflet, but no discernible microcapsular layer. Optimally cultivated in cell culture (Vero, $X$. laevis, and tick cell lines [Yunker and Cory, submitted for publication]). Many strains give rise to plaques and a characteristic cytopathic effect in Vero cells, which distinguishes them from other plaque-forming rickettsiae. An obligate intracellular parasite. Replication by binary fission occurs primarily in the cytoplasm and rarely in the nuclei of host cells. Nonpathogenic or of low pathogenicity for chicken embryos when the yolk sac route of inoculation is used. A few strains can be established in chicken embryos, but growth of rickettsiae in yolk sacs is never luxuriant. Nonpathogenic for randomly bred adult white Swiss mice, guinea pigs, and $M$. pennsylvanicus. Limited replication of some strains may occur in voles, but these strains cannot be maintained by serial passage in this vertebrate. Cells possess surface antigens that cross-react with convalescent sera from Rocky Mountain spotted fever, epidemic typhus, and murine typhus patients, as determined by indirect immunofluorescence. Cross-reactions may also occur with antisera prepared in guinea pigs, rabbits, and goats against $R$. rickettsii, but not in randomly bred white Swiss mice. Protein composition as determined by SDS-PAGE is unlike the protein compositions of TG and SFG rickettsiae. The $\mathrm{G}+\mathrm{C}$ content of the DNA is approximately $30 \mathrm{~mol} \%$ (as determined by thermal denaturation), a level similar to the $\mathrm{G}+\mathrm{C}$ content of TG rickettsiae but lower than the $\mathrm{G}+\mathrm{C}$ content of SFG organisms. The type strain, RML 369-C, is held in The Rocky Mountain Laboratories Collection.

At present, there is no evidence that rickettsiae of the $R$. bellii serogroup cause human illness (Philip and Casper, manuscript in preparation). Nothing is known about the mechanism for maintenance of $R$. bellii in nature except that strains of the $R$. bellii serogroup have been recovered from two argasid and six ixodid tick species from various regions of the United States and that transovarial transmission and transstadial transmission of these organisms have been demonstrated in $D$. andersoni.

\section{ACKNOWLEDGMENTS}

We are grateful to all those who provided many of the rickettsial strains upon which this study was based. We particularly thank Robert S. Lane (University of California,
Berkeley), J. Newton MacCormack (North Carolina Department of Human Resources, Raleigh), and Scott W. Gordon (Ohio Department of Health, Columbus) for collecting ticks in their states from which rickettsiae were isolated. We also thank Richard A. Ormsbee, Hamilton, Mont., for kindly providing the convalescent sera originally obtained by James Plorde, Seattle, Wash., from epidemic typhus patients. James Whitlock, Raymond Mann, and Anthony Mavros gave excellent technical support. We are appreciative above all to $\mathrm{E}$. John Bell, Hamilton, Mont., who provided the $R$. bellii prototype, information pertaining to it, and impetus for this study.

\section{LITERATURE CITED}

1. Anacker, R. L., T. F. McCaul, W. Burgdorfer, and R. K. Gerloff. 1980. Properties of selected rickettsiae of the spotted fever group. Infect. Immun. 27:468-474.

2. Burgdorfer, W. 1970 . Hemolymph test. A technique for detection of rickettsiae in ticks. Am. J. Trop. Med. Hyg. 19:1010-1014.

3. Cory, J., C. E. Yunker, J. A. Howarth, Y. Hokama, L. E. Hughes, L. A. Thomas, and C. M. Clifford. 1975. Isolation of spotted fever group and Wolbachia-like agents from field-collected materials by means of plaque formation in mammalian and mosquito cells. Acta Virol. 19:443-445.

4. Hayes, S. F., and W. Burgdorfer. 1979. Uitrastructure of Rickettsia rhipicephali, a new member of the spotted fever group rickettsiae in tissues of the host vector Rhipicephalus sanguineus. J. Bacteriol. 137:605-613.

5. Lackman, D. B., E. J. Bell, H. G. Stoenner, and E. G. Pickens. 1965. The Rocky Mountain spotted fever group of rickettsias. Health Lab. Sci. 2:135-141.

6. Laemmli, U. K. 1970 . Cleavage of structural proteins during the assembly of the head of bacteriophage T4. Nature (London) 227:680-685.

7. Lane, R. S., R. N. Philip, and E. A. Casper. 1981. Ecology of tick-borne agents in California. II. Further observations on rickettsiae, p. 575-584. In W. Burgdorfer and R. L. Anacker (ed.), Rickettsiae and rickettsial diseases. Academic Press, Inc., New York.

8. Marmur, J., and P. Doty. 1962. Determination of the base composition of deoxyribonucleic acid from its thermal denaturation temperature. J. Mol. Biol. 5:109-118.

9. McKiel, J. A., E. J. Bell, and D. B. Lackman. 1967. Rickettsia canada: a new member of the typhus group of rickettsiae isolated from Haemaphysalis leporispalustris ticks in Canada. Can. J. Microbiol. 13:503-510.

10. Myers, W. F., and C. L. Wisseman, Jr. 1980. Genetic relatedness among the typhus group of rickettsiae. Int. J. Syst. Bacteriol. 30:143-150.

11. Myers, W. F., and C. L. Wisseman, Jr. 1981. The taxonomic relationship of Rickettsia canada to the typhus and spotted fever groups of the genus Rickettsia, p. 313-326. In W. Burgdorfer and R. L. Anacker (ed.), Rickettsiae and rickettsial diseases. Academic Press, Inc., New York.

12. Philip, R. N., and E. A. Casper. 1981. Serotypes of spotted fever group rickettsiae isolated from Dermacentor andersoni (Stiles) ticks in western Montana. Am. J. Trop. Med. Hyg. 30:230-238.

13. Philip, R. N., E. A. Casper, W. Burgdorfer, R. K. Gerloff, L. E. Hughes, and E. J. Bell. 1978. Serologic typing of rickettsiae of the spotted fever group by microimmunofluorescence. J. Immunol. 121:1961-1968.

14. Philip, R. N., E. A. Casper, R. A. Ormsbee, M. G. Peacock, and W. Burgdorfer. 1976. Microimmunofluorescence test for the serological study of Rocky Mountain spotted fever and typhus. J. Clin. Microbiol. 3:51-61.

15. Philip, R. N., R. S. Lane, and E. A. Casper. 1981. Serotypes of tick-borne spotted fever group rickettsiae from western California. Am. J. Trop. Med. Hyg. 30:722-727.

16. Silverman, D. J., and C. L. Wisseman, Jr. 1978. Comparative ultrastructural study of the cell envelopes of Rickettsia prowazekii, Rickettsia rickettsii, and Rickettsia tsutsu- 
gamushi. Infect. Immun. 21:1020-1023.

17. Silverman, D. J., C. L. Wisseman, Jr., A. D. Waddell, and M. Jones. 1978. External layers of Rickettsia prowazekii and Rickettsia rickettsii: occurrence of a slime layer. Infect. Immun. 22:233-246.

18. Stoenner, H. G., D. B. Lackman, and E. J. Bell. 1962. Factors affecting the growth of rickettsias of the spotted fever group in fertile hens' eggs. J. Infect. Dis. 110:121128.

19. Tyeryar, F. J., Jr., E. Weiss, D. B. Millar, F. M. Boze- man, and R. A. Ormsbee. 1973. DNA base composition of rickettsiae. Science 180:415-417.

20. Weiss, E., J. C. Coolbaugh, and J. C. Williams. 1975. Separation of viable Rickettsia typhi from yolk sac and Lcell host components by Renografin density gradient centrifugation. Appl. Microbiol. 30:456-463.

21. Wisseman, C. L., Jr., A. D. Waddell, and W. T. Walsh. 1974. In vitro studies of the action of antibiotics on Rickettsia prowazeki by two basic methods of cell culture. J. Infect. Dis. 130:564-573. 